Check for updates

Cite this: RSC Adv., 2017, 7, 27449

Received 19th March 2017

Accepted 15th May 2017

DOI: 10.1039/c7ra03214e

rsc.li/rsc-advances

\title{
A new light triggered approach to develop a micro porous tough hydrogel $\dagger$
}

\author{
Qingqing Dou, t $^{\mathrm{a}}$ Zhi Wei Kenny Low, (D) t $^{\mathrm{ab}}$ Kangyi Zhang ${ }^{\mathrm{a}}$ and Xian Jun Loh (D) *abc \\ A porous tough hydrogel (alginate-polyacrylamide) is successfully synthesized using a photo-gelling \\ polymer as a templating agent. Unlike current porous hydrogels, we present a useful pore-enhancing \\ strategy without sacrificing the mechanical properties of the parent matrix. These porous tough \\ hydrogels, as 3D cell culture scaffolds, can mimic the extracellular matrix and allow maximum nutrient \\ exchange.
}

\section{Introduction}

Conventional cell culture is carried out on two-dimensional (2D) substrates, such as cell culture flasks or Petri dishes. Cells cultured using such methods fail to simulate actual cell behavior in the body in terms of morphology, biomarker expression and cell signalling etc. ${ }^{1}$ To maintain cell morphology and provide mechanical support like an extracellular matrix (ECM), scaffolds for 3D cell culture are required. 3D cell cultures can be developed for the purpose of artificial organs, bioprinting and cell therapy. Currently, several types of materials are available - silk fibroin,${ }^{2-4}$ porcine meniscus-derived hydrogels, ${ }^{5}$ synthetic collagen, ${ }^{6}$ and polymeric hydrogels. ${ }^{7}$ A porous polymeric hydrogel is advantageous because of the high specific surface area with high flux, ease of fabrication, and property tunability. ${ }^{8}$ Available methods used to generate foam hydrogels include gas foaming/salt leaching, solvent casting/particle leaching, thermally-induced phase separation, melt molding, emulsion and freeze-drying. ${ }^{9-17}$ Electrospinning has also been used to generate ECM-like structures for cell culture. ${ }^{18-25}$ However, non-uniformity of pores or lack of tunability may occur due to porogen sedimentation, limited options for particle size, poor thermal conduction and phase separation, difficulty in porogen leaching and instability of the emulsion. On the other hand, the high water content of these hydrogels makes them mechanically weak and inherently unsuitable for artificial muscle and cartilage. ${ }^{26}$ Tough hydrogels have been developed to overcome the weaknesses of these materials..$^{27-29}$

\footnotetext{
${ }^{a}$ Institute of Materials Research and Engineering (IMRE), 2 Fusionopolis Way, \#08-03. Innovis, Singapore 138634. E-mail: lohxj@imre.a-star.edu.sg

${ }^{b}$ Department of Materials Science and Engineering, National University of Singapore, 9 Engineering Drive 1, Singapore 117576, Singapore

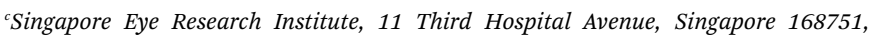
Singapore

$\dagger$ Electronic supplementary information (ESI) available. See DOI: 10.1039/c7ra03214e

‡ Co-first author.
}

Suo et $a .^{30}$ developed alginate-polyacrylamide (ALG-PAAm) interpenetrating network hydrogels of $90 \%$ water content with high mechanical strength and stretchability. The combination of naturally-derived ALG and bio-inert PAAm makes the scaffold cell-compatible. The superior mechanical property of ALGPAAm, with a fracture toughness of $\sim 9000 \mathrm{~J} \mathrm{~m}^{-2},{ }^{31}$ enables mechanical support similar to muscle tissue..$^{32}$ ALG-PAAm can resist mechanical degradation in cell culture conditions and show minimal side effects on cells in vitro and in vivo. ${ }^{31}$ However, these cell studies have largely been limited to the surface of the hydrogel. The generation of 3D pores in the cell culture substrate is essential for tissue engineering and wound healing because cells migrate in all directions. Hence, a porous scaffold can improve the bulk integration with surrounding tissue in the implanted host. The main challenge of current porogen leaching method is to minimize weakening of mechanical properties while increasing pore size.

Here, we propose a porous ALG-PAAm hydrogel with controllable pore size using a top-down method. EPS, a spiropyran-based light responsive polymer developed by our group recently, ${ }^{33}$ is used as a templating agent. When exposed to an incident wavelength of $535 \mathrm{~nm}$, EPS chains collapse and undergo a reversible sol-gel transition. Thus, EPS gelation and photo-polymerization of the chemical network in ALG/PAAm can occur simultaneously via exposure to a broad spectrum UV light source. By causing the EPS gels to revert back into the sol state, vacancies are left in the hydrogel network. There are several parameters which can be adjusted to tune the properties. The pore size can be controlled by the amount of EPS used. The toughness of the scaffold can be controlled by factors such as cationic soaking solution concentration, the crosslinker ratio and UV irradiation intensity. Thus, the flexibility of this scaffold design enables it to mimic muscle, heart valves and other organs. This design shows three advantages for fabricating scaffolds for 3D cell culture. Firstly, the procedure for obtaining porous hydrogel is relatively easier compared to other methods such as lithography. ${ }^{34}$ Pore size enhancement is achieved 
through light exposure and immersion in water. The facile nature of this method avoids the production of any contaminants or toxins within the gel. Secondly, a high water content hydrogel with controllable pore volume and number allows maximum oxygen and carbon dioxide exchange, providing cells with a favorable environment for growth and proliferation. Thirdly, mechanical strength can be easily tuned to mimic different organs and functions. Our system presents high potential for tissue-mimicking phantoms and implantable tissue.

\section{Results and discussion}

The process of making the 3D scaffold is shown in Fig. 1. The ALG-PAAm hydrogels are synthesized by modifying the methods previously developed by Suo et $a l^{.30,35}$ The light responsive polymer EPS is mixed with the precursors of alginate-polyacrylamide (ALG-PAAm) hydrogel before UV curing. UV exposure using a mercury vapour lamp induces polymerization of acrylamide monomers, and simultaneously causes gelation of EPS. The EPS thus forms gel clusters which within the ALG-PAAm matrix. The as-synthesized hydrogel is then soaked in $\mathrm{CaCl}_{2}$ solution to form the ionic ALG-Ca ${ }^{2+}$ network. Subsequently, the hydrogel is transferred into DI water and exposed to sunlight. The EPS gel undergoes gel-to-sol transition and diffuses out of the network leaving vacancies for cell growth and proliferation. The complete removal of EPS is ensured by washing the prepared gels in DI water thoroughly.

Due to a lack of compatible crosslinking sites with ALGPAAm, the EPS clusters should leach out of the network upon rinsing, without altering the chemical properties of ALG-PAAm. The vacancies left by EPS are then replaced with water during washing, resulting in the larger pore sizes observed. The hydrogels were cast in cylindrical moulds for easier characterization. To show the flexibility of this method, the loading concentration of EPS is varied and four types of scaffolds were fabricated $-0,5,10$ and $30 \mathrm{wt} \%$. Samples are subsequently named Ex, where $x$ represents the weight concentration of EPS added to the precursor solution.

The morphology of each gel is studied under SEM. Gel samples are instantly frozen with liquid nitrogen to maintain

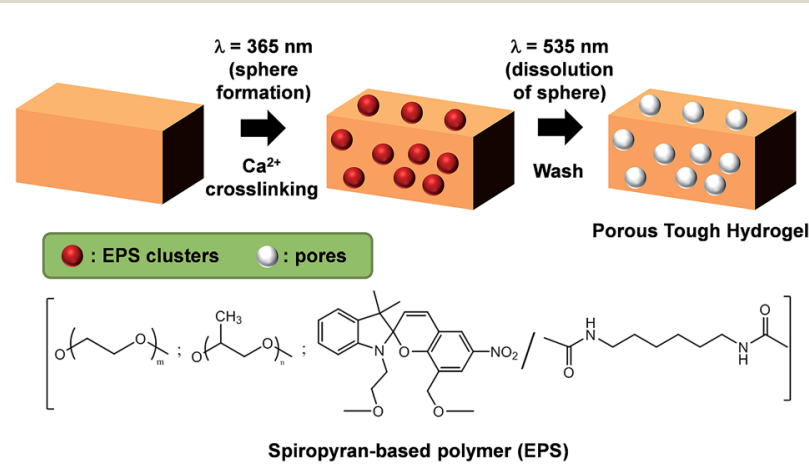

Fig. 1 Method to fabricate porous tough hydrogels as 3D cell culture scaffold and the structure of the spiropyran-based polymer (EPS) used for templating. the original pore size and volume, and subsequently freezedried. The SEM images clearly show that pore size in the gel increases with increasing amounts of EPS used for synthesis (Fig. 2). In the absence of EPS, the average pore size in E0 lies around the $5 \mu \mathrm{m}$ range. On the other hand, the addition of EPS causes pore size enhancement in the gels. At $5 \mathrm{wt} \%$ of EPS, pore size increases to $30-40 \mu \mathrm{m}$, while $10 \%$ addition in E10 results in pore sizes of up to $70 \mu \mathrm{m}$. At the same time, this is accompanied by a decrease in the number of pores in the same area of interest. Significantly larger pore size is also observed in E30 (Fig. S1†). However the E30 gels are extremely weak and hence excluded from subsequent experiments. This agrees with previous studies whereby increases in degree of porosity have a negative effect on overall mechanical properties. ${ }^{36,37}$ In this case, the large number of large pores results in a lower polymer volume density, resulting in thinner network walls with low mechanical strength. Thus, the percentage of EPS used as template should not be too high.

Pore enhancement occurs due to EPS leaving the gel network after soaking. Due to the lack of active crosslinking sites in EPS, the templating agent is not chemically bonded to the network. EPS thus leaches out of the gel upon re-solubilization. This phenomenon is confirmed by TGA analysis. As seen from the differential TGA curves in Fig. $\mathrm{S} 2, \uparrow$ the thermal degradation of EPS occurs in a single step at $409{ }^{\circ} \mathrm{C}$. This coincides with the degradation profiles of PEO-PPO copolymers such as Pluronic. $^{38}$ This is expected, since the structure of EPS largely consists of a PEO-PPO-PEO tri-block structure. On the other hand, the thermal degradation profiles of lyophilized EPSdoped hydrogels, show two main degradation steps at $270{ }^{\circ} \mathrm{C}$ and $358{ }^{\circ} \mathrm{C}$. The lack of a corresponding weight loss peak at $409{ }^{\circ} \mathrm{C}$ hence implies the absence of EPS in the gels.

High water absorption is essential for cell scaffolds to retain nutrition and drugs - a behaviour similar to the extracellular matrix (ECM). Lyophilized gels are re-immersed in water to determine their water re-absorption ability through weight measurements. The gels are found to absorb water equivalent to $21.73 \pm 0.41,25.23 \pm 1.81$ and $30.62 \pm 5.71$ times their respective dry weights for E0, E5 and E10. The EPS templated porous hydrogels are shown to have a higher capacity for water absorption. The re-absorbed water contents are slightly higher than the initial equilibrium water contents of the gels. This is likely due to the ionic groups ${ }^{39}$ scattered within the threedimensional capillary network of polyacrylamide. Favourable interactions such as ionic attraction, osmotic pressure ${ }^{40}$ and capillary forces promote water uptake. ${ }^{\mathbf{4 1}}$ Larger pores increase

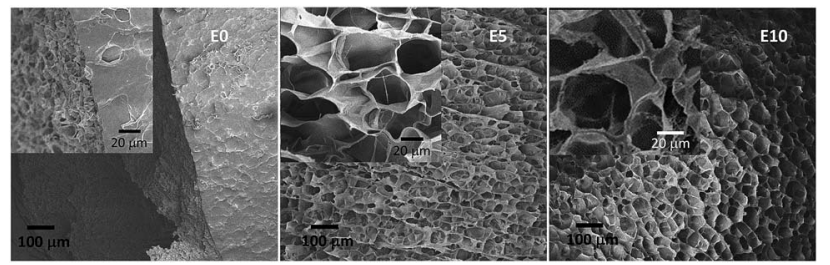

Fig. 2 SEM images of ALG-PAAm hydrogels UV cured with $0 \%, 5 \%$ and $10 \%$ of EPS pore-template. 
surface area and promote water uptake ability due to greater ion exposure. Additionally, enhanced pore sizes decrease the density of polymer within the gel and increase the water content.

A hydrogel scaffold with implantable potential should show good mechanical tolerance. The mechanical properties of the EPS-templated gels are evaluated by unconfined uniaxial compression tests. Our results show that the increase of porosity has minimal effect on the load-bearing ability of the gels (Fig. S3 $\dagger$ ). The properties remain within the statistical range of properties of the control gel E0 (Table 1). Compressive moduli for all gels remain within the range of 90-130 kPa. Similarly, the strength at $80 \%$ strain is found to be $200-300 \mathrm{kPa}$. It is worth highlighting that the gels are still robust. Throughout all tests, no sign of mechanical rupture is observed in the gels despite the high degree of mechanical strain. Thus the hydrogel can totally support its compression stress upon implantation without the risk of complete failure. The measured moduli values are comparable to that of other chemically-crosslinked tough hydrogels. ${ }^{\mathbf{4 2 - 4 4}}$ Additionally, such mechanical properties are similar to that of human tissue, especially those within the 1-100 $\mathrm{kPa}$ compressive modulus range. ${ }^{45-48}$ The compressive toughness of the gels remains within the statistical range of 40$60 \mathrm{~kJ} \mathrm{~m}^{-3}$, showing that the altered pore sizes do not change the amount of energy absorbed during deformation. While average pore size has increased, the overall degree of porosity in the hydrogel remains approximately constant. This can be seen by the minimal deviation in water content of the templated gels.

The mechanical property of the hydrogels was further determined with a rotational rheometer under a strain of $1 \%$ and at the frequency of $1 \mathrm{~Hz}$. The storage modulus was found to be in the range of 2000-3500 Pa (Fig. S4 $\dagger$ ). Hydrogels of PEG-PA (storage modulus $\sim 500 \mathrm{~Pa})^{1}$ and PLX-PA and PA-PLX-PA/HA (storage modulus 400-1600 $\mathrm{Pa})^{49}$ have been reported for 3D cell culture as well. However, these materials form hydrogel at higher concentrations and the pore sizes are also smaller. The enhancement of storage modulus for $10 \%$ (Fig. S4†) does not conflict with the above compression test, as the storage modulus is regarded as inherent strength.

We show that any difference in mechanical property between our templated gels and control gels is minimal. As mentioned above, an increase in degree of porosity can weaken a hydrogel, which leads us to conclude that pore size increases while the polymer volume density in the network remains constant. Thus the EPS-templated gels have lesser pores of larger volumes. This thus does not change the load-bearing ability of the porous hydrogel. This also confirms our hypothesis that EPS does not form crosslinks with the alginate and PAAm networks, and instead leaches out of the hydrogel matrix during the long immersion step. While changes in macroscopic physical properties are minimized, we observe that the reported pore sizes are similar to cellular dimension. ${ }^{50}$ These templated hydrogels can thus influence cellular growth.

To investigate any potential toxic side-effects of using EPS as a templating agent, biocompatibility of various scaffolds was assessed using CellTiter-Blue ${ }^{\circledR}$ assay (Promega) and live-dead staining (FDA fluorescein diacetate/PI propidium iodide). Prior to testing, gel samples are treated with ethanol for purification and disinfection, followed by a thorough rinsing in PBS. NIH/3T3 fibroblasts were cultured on the hydrogels for 14 days to allow cell proliferation. Cell viability test show the hydrogels remained non-toxic and compatible to the cells, and instead promote additional cell proliferation over a 14 day period. The highest cell density was observed in E10 (Fig. 3). Cell viability of this scaffold is significantly different from that of E0 $(* p<0.05)$ and the control scaffold without cell $(* * * p<0.0005)$. The results show that scaffold with larger pores accommodates more cells. While the mean cell viability for both E5 and E0 scaffolds are higher than the control scaffold, the differences are insignificant. Longer cultivation period should result in significance for E5 and E0. Additionally, good biocompatibility is observed for E5 and E10 based on live-dead fluorescent micrographs at day 28 (Fig. 4). The fibroblasts present their characteristic elongated, spindle-like morphology. Both E5 and E10 EPS-templated scaffolds show few dead cells amidst a majority of live cells. Without EPS doping, a large proportion of fibroblasts fail to survive. This may be due to the lack of oxygen/carbon dioxide exchange and poor nutrition/waste exchange in the low porosity scaffold.

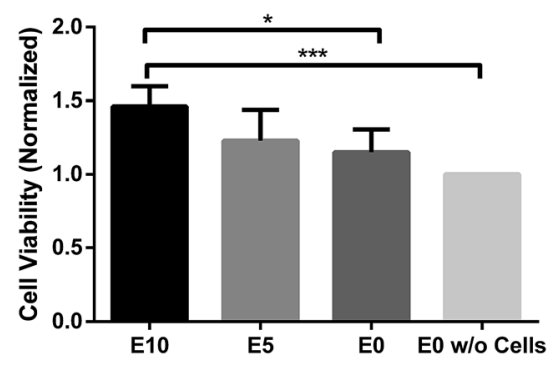

Fig. 3 Cell viability of NIH/3T3 fibroblasts after 14 days of growth on various scaffolds as determined by fluorometric resazurin assay (CellTiter-Blue ${ }^{\circledR}$ ). Fluorescence readings have been normalized to the control scaffold (EO w/o cells). (EO: Alg-AMM cultured with cells; EO w/o cells (control): Alg-AMM without cell seeding; $N=3, * p<0.05$, $* * * p<0.0005)$

Table 1 Compressive modulus and strength of samples

\begin{tabular}{llll}
\hline $\begin{array}{l}\text { EPS templated } \\
\text { content }\end{array}$ & $\begin{array}{l}\text { Compressive modulus } \\
(\mathrm{kPa})\end{array}$ & $\begin{array}{l}\text { Strength at 80\% } \\
\text { strain (kPa) }\end{array}$ & $\begin{array}{l}\text { Compressive toughness } \\
\left(\mathrm{kJ} \mathrm{m} \mathrm{m}^{-3}\right)\end{array}$ \\
\hline E0 & $116 \pm 37$ & $215 \pm 52$ & $45.2 \pm 16$ \\
E5 & $126 \pm 19$ & $278 \pm 43$ & $53.8 \pm 10$ \\
E10 & $97.5 \pm 13$ & $281 \pm 94$ & $47.8 \pm 11$
\end{tabular}




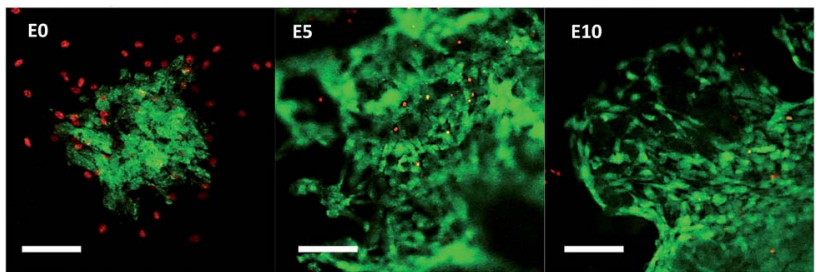

Fig. 4 Fluorescent images of fibroblasts showing the proportion of live cells (green) to dead cells (red) on various scaffolds. The cells were stained with FDA (green) and PI (red) after 28 days of incubation on the scaffold (scale bar is $100 \mu \mathrm{m}$ ). Viable cell cytoplasm would be labelled green, while non-viable cell nuclei would be labelled red.

\section{Conclusion}

In this work, we design a biocompatible porous hydrogel for 3D cell culture using ALG-PAAm interpenetrating networks. The pores are incorporated during UV curing stage by using our previously reported light responsive polymer EPS. Exposure to UV light causes different responses in ALG-PAAm and EPS. The chemical network in ALG-PAAm forms permanently, while EPS undergoes a sol-gel transition within the chemical network. By simply soaking in water, EPS can be removed completely, leaving pores in the micron scale in ALG-PAAm. There is no significant negative impact on the mechanical properties (up to $30 \%$ of EPS template) making these tough hydrogels suitable for implantation. Porosity further improves cell viability as seen from NIH/3T3 fibroblast culture. The effect of pore enhancement on biodegradation will be explored in future. Our method provides a new way to make porous hydrogels, and this can be applied to other materials as well.

\section{Experimental}

\section{Synthesis of hydrogel for scaffold}

Alginate, EPS and acrylamide were completely dissolved in DI water. The solution was purged using $\mathrm{N}_{2}$ gas for 1 hour, before $N, N^{\prime}$-methylenebis(acrylamide) (BIS) covalent crosslinker and ammonium persulfate (APS) photoinitiator were added. The prepolymer solution was then poured into cylindrical moulds and cured with a mercury vapour light source $(254 \mathrm{~nm}, 50 \mu \mathrm{W}$ $\mathrm{cm}^{-2}$ ) for 2 hours. The polymerized gels were then immersed in $5 \mathrm{M} \mathrm{CaCl}_{2}$ solution for 2 days to allow calcium alginate crosslinking to occur, before being immersed in DI water for 1 week. A detailed account on hydrogel preparation has been previously reported by our group. ${ }^{35}$

\section{Characterization}

Soaked gel samples were weighed daily. When the soaked gel weight did not differ by more than $1 \%$ from that of the previous day, equilibrium swelling was deemed to have occurred. The water content in the gels was determined by freeze-drying.

\section{Scanning electron microscopy (SEM)}

Morphology of the scaffold was determined using JEOL FESEM JSM6700F. Samples were frozen in liquid nitrogen, sliced into thin pieces $(\sim 1 \mathrm{~mm})$ and dried in vacuo. Gold spraying was performed to enhance the conductivity of samples. The samples were then viewed under accelerating voltage $5 \mathrm{kV}$ with $8.0 \mathrm{~mm}$ working distance.

\section{Thermogravimetric analysis (TGA)}

Thermal degradation profiles of freeze-dried gels were studied using a TGA Q500 (TA Instruments) under a nitrogen atmosphere and heating rate of $20{ }^{\circ} \mathrm{C} \mathrm{min}{ }^{-1}$. Samples were tested from 150 to $600{ }^{\circ} \mathrm{C}$ on an alumina pan.

\section{Rehydration studies}

The rehydration of gels was observed through gravimetric analysis. Lyophilized gels were first measured to determine the dry weight $\left(W_{\mathrm{d}}\right)$ before being immersed in excess DI water. The weight of the respective samples was monitored on a daily basis until equilibrium $\left(W_{\mathrm{eq}, 2}\right)$. Finally, the water re-absorption ability is described by the equilibrium swelling ratio of rehydrated gels.

$$
\text { Equilibrium swelling ratio }=\frac{\left(W_{\mathrm{eq}, 2}-W_{\mathrm{d}}\right)}{W_{\mathrm{d}}}
$$

\section{Mechanical test}

Uniaxial compression test was done using an Instron 5543 mechanical testing machine at a strain rate of $1 \mathrm{~mm} \mathrm{~min}^{-1}$. Prior to testing, hydrogel samples were first immersed in deionized water for 7 days in order to obtain equilibrium swelling. $1 \mathrm{kN}$ load cell was used for all measurements, and compression was done to a compressive strain of $95 \%$.

\section{Rheological test}

The hydrogel was cast in a $3 \mathrm{ml}$ diameter cylinder and sliced into a thin layer of about $1 \mathrm{~mm}$ for rheology test. The sample was placed horizontally in between two parallel plates and an initial $2 \mathrm{~N}$ axial force was normally applied to it. The axial force was monitored when the scaffold was compressed by the head at a speed of $1.0 \mu \mathrm{m} \mathrm{s}^{-1}$ without angular rotation.

\section{Biocompatibility testing}

Hydrogels were cleaned using $70 \mathrm{vol} \%$ ethanol followed by water rinsing. The scaffolds were then soaked in DMEM (ATCC Dulbecco's Modified Eagle's Medium) overnight in a $37^{\circ} \mathrm{C}$ cell incubator. Before the cells were grown on the scaffolds, the gels were also UV sterilized. The hydrogels were shaken for $2 \mathrm{~h}$ in $50 \mathrm{ml}$ falcon tubes containing NIH/3T3 cell suspension $\left(1 \times 10^{6}\right.$ cells per ml ATCC ${ }^{\circledR}$ CRL- $\left.1658^{\mathrm{TM}}\right)$. The scaffolds were rinsed in PBS to remove unattached cells. Next the scaffolds were transferred to 12-well plates for cell cultivation. Culture medium was replaced every two days. After 14 days, resazurin cell viability assay (CellTiter-Blue ${ }^{\circledR}$ Promega) was performed. Fluorescence readings were normalized to a control scaffold without cell. The scaffolds were rinsed three times with PBS before resuming cell culture. At day 28, live-dead staining was performed using FDA/ 
PI (fluorescein diacetate/propidium iodide (Sigma-Aldrich)) and cells were visualized under a microscope (Leica DM5000 B).

\section{Notes and references}

1 M. H. Park, H. J. Moon, J. H. Park, U. P. Shinde, D. Y. Ko and B. Jeong, Macromol. Biosci., 2015, 15, 464-472.

2 Y. Wang, U.-J. Kim, D. J. Blasioli, H.-J. Kim and D. L. Kaplan, Biomaterials, 2005, 26, 7082-7094.

3 B. B. Mandal and S. C. Kundu, Biomaterials, 2009, 30, 29562965.

4 A. Woloszyk, S. H. Dircksen, N. Bostanci, R. Müller, S. Hofmann and T. A. Mitsiadis, PLoS One, 2014, 9, e111010. 5 J. Wu, Q. Ding, A. Dutta, Y. Wang, Y.-h. Huang, H. Weng, L. Tang and Y. Hong, Acta Biomater., 2015, 16, 49-59.

6 Z. Luo, Y. Yue, Y. Zhang, X. Yuan, J. Gong, L. Wang, B. He, Z. Liu, Y. Sun and J. Liu, Biomaterials, 2013, 34, 4902-4913.

7 D. Seliktar, Science, 2012, 336, 1124-1128.

8 W. S. Toh and X. J. Loh, Mater. Sci. Eng., C, 2014, 45, 690-697. 9 H. Janik and M. Marzec, Mater. Sci. Eng., C, 2015, 48, 586591.

10 M. Alizadeh, F. Abbasi, A. B. Khoshfetrat and H. Ghaleh, Mater. Sci. Eng., C, 2013, 33, 3958-3967.

11 Y. S. Cho, M. W. Hong, S.-Y. Kim, S.-J. Lee, J. H. Lee, Y. Y. Kim and Y.-S. Cho, Mater. Sci. Eng., C, 2014, 45, 546-555.

12 A. Díaz Lantada, H. Alarcón Iniesta and J. P. García-Ruíz, Mater. Sci. Eng., C, 2016, 59, 218-227.

13 M. Flaibani and N. Elvassore, Mater. Sci. Eng., C, 2012, 32, 1632-1639.

14 J. Mao, S. Duan, A. Song, Q. Cai, X. Deng and X. Yang, Mater. Sci. Eng., C, 2012, 32, 1407-1414.

15 S. Mohanty, K. Sanger, A. Heiskanen, J. Trifol, P. Szabo, M. Dufva, J. Emnéus and A. Wolff, Mater. Sci. Eng., C, 2016, 61, 180-189.

16 A. R. Siddiq and A. R. Kennedy, Mater. Sci. Eng., C, 2015, 47, 180-188.

17 D. Sin, X. Miao, G. Liu, F. Wei, G. Chadwick, C. Yan and T. Friis, Mater. Sci. Eng., C, 2010, 30, 78-85.

18 D. Kai, W. Ren, L. Tian, P. L. Chee, Y. Liu, S. Ramakrishna and X. J. Loh, ACS Sustainable Chem. Eng., 2016, 5268-5276.

19 D. Kai, M. P. Prabhakaran, B. Q. Chan, S. S. Liow, S. Ramakrishna, F. Xu and X. J. Loh, Biomed. Mater., 2016, 11, 015007.

20 D. Kai, M. J. Tan, M. P. Prabhakaran, B. Q. Chan, S. S. Liow, S. Ramakrishna and X. J. Loh, Colloids Surf., B, 2016, 148, 557-565.

21 R. Lakshminarayanan, R. Sridhar, X. J. Loh, M. Nandhakumar, V. A. Barathi, M. Kalaipriya, J. L. Kwan, S. P. Liu, R. W. Beuerman and S. Ramakrishna, Int. J. Nanomed., 2014, 9, 2439-2458.

22 X. J. Loh, P. Peh, S. Liao, C. Sng and J. Li, J. Controlled Release, 2010, 143, 175-182.

23 D. Kai, S. Jiang, Z. W. Low and X. J. Loh, J. Mater. Chem. B, 2015, 3, 6194-6204.

24 J. Hu, D. Kai, H. Ye, L. Tian, X. Ding, S. Ramakrishna and X. J. Loh, Mater. Sci. Eng., C, 2017, 70, 1089-1094.
25 D. Kai, S. S. Liow and X. J. Loh, Mater. Sci. Eng., C, 2014, 45, 659-670.

26 P. Thoniyot, M. J. Tan, A. A. Karim, D. J. Young and X. J. Loh, Adv. Sci., 2015, 2, 1400010.

27 D. Rana and M. Ramalingam, Mater. Sci. Eng., C, 2017, 76, 1057-1065.

28 M. Sabzi, N. Samadi, F. Abbasi, G. R. Mahdavinia and M. Babaahmadi, Mater. Sci. Eng., C, 2017, 74, 374-381.

29 K. Tonsomboon, A. L. Butcher and M. L. Oyen, Mater. Sci. Eng., C, 2017, 72, 220-227.

30 J.-Y. Sun, X. Zhao, W. R. K. Illeperuma, O. Chaudhuri, K. H. Oh, D. J. Mooney, J. J. Vlassak and Z. Suo, Nature, 2012, 489, 133-136.

31 M. C. Darnell, J.-Y. Sun, M. Mehta, C. Johnson, P. R. Arany, Z. Suo and D. J. Mooney, Biomaterials, 2013, 34, 8042-8048.

32 M. M. Fitzgerald, K. Bootsma, J. A. Berberich and J. L. Sparks, Biomacromolecules, 2015, 16, 1497-1505.

33 Q. Dou, S. S. Liow, W. Weng and X. J. Loh, J. Polym. Sci., Part A: Polym. Chem., 2016, 54, 2837-2844.

34 G. Wei and P. X. Ma, J. Biomed. Mater. Res., Part A, 2006, 78, 306-315.

35 Z. W. Low, P. L. Chee, D. Kai and X. J. Loh, RSC Adv., 2015, 5, 57678-57685.

36 S. M. LaNasa, I. T. Hoffecker and S. J. Bryant, J. Biomed. Mater. Res., Part B, 2011, 96, 294-302.

37 Y.-C. Chiu, S. Kocagöz, J. C. Larson and E. M. Brey, PLoS One, 2013, 8, e60728.

38 T. Wang, Y. Wu and A. J. Zeng, J. Appl. Polym. Sci., 2010, 117, 604-613.

39 D. R. Heldman, Encyclopedia of Agricultural, Food, and Biological Engineering (Print), CRC Press, 2003.

40 D. Li, X. Zhang, J. Yao, G. P. Simon and H. Wang, Chem. Commun., 2011, 47, 1710-1712.

41 F. Hua and M. Qian, J. Mater. Sci., 2001, 36, 731-738.

42 Y. Sun, S. Liu, G. Du, G. Gao and J. Fu, Chem. Commun., 2015, 51, 8512-8515.

43 M. Bartnikowski, R. Wellard, M. Woodruff and T. Klein, Polymers, 2015, 7, 1539.

44 E. C. Muniz and G. Geuskens, Macromolecules, 2001, 34, 4480-4484.

45 R. M. Delaine-Smith, S. Burney, F. R. Balkwill and M. M. Knight, J. Mech. Behav. Biomed. Mater., 2016, 60, 401-415.

46 M. S. Detamore and K. A. Athanasiou, J. Oral Maxillofac. Surg., 2003, 61, 494-506.

47 K. Holst, H. Liebgott, J. E. Wilhjelm, S. Nikolov, S. T. TorpPedersen, P. Delachartre and J. A. Jensen, Ultrasonics, 2013, 53, 439-446.

48 R. K. Korhonen, M. S. Laasanen, J. Töyräs, J. Rieppo, J. Hirvonen, H. J. Helminen and J. S. Jurvelin, J. Biomech., 2002, 35, 903-909.

49 M. H. Park, B. G. Choi and B. Jeong, Adv. Funct. Mater., 2012, 22, 5118-5125.

50 B. Alberts, A. Johnson, J. Lewis, D. Morgan, M. Raff, K. Roberts and P. Walter, Molecular Biology of the Cell, Taylor \& Francis Group, 6th edn, 2014. 